\title{
Data Analysis for the Aero Derivative Engines Bleed System Failure Identification and Prediction
}

\author{
Khalid Salmanov \\ Engineering Institute of Technology, Perth, WA 6005, Australia \\ E-mail: 5949@student.eit.edu.au \\ Hadi Harb \\ Engineering Institute of Technology, Perth, WA 6005, Australia \\ E-mail: hadi.harb@eit.edu.au
}

Received: 18 August 2021; Accepted: 06 October 2021; Published: 08 December 2021

\begin{abstract}
Middle size gas/diesel aero-derivative power generation engines are widely used on various industrial plants in the oil and gas industry. Bleed of Valve (BOV) system failure is one of the failure mechanisms of these engines. The $\mathrm{BOV}$ is part of the critical anti-surge system and this kind of failure is almost impossible to identify while the engine is in operation. If the engine operates with BOV system impaired, this leads to the high maintenance cost during overhaul, increased emission rate, fuel consumption and loss in the efficiency. This paper proposes the use of readily available sensor data in a Supervisory Control and Data Acquisition (SCADA) system in combination with a machine learning algorithm for early identification of BOV system failure. Different machine learning algorithms and dimensionality reduction techniques are evaluated on real world engine data. The experimental results show that Bleed of Valve systems failures could be effectively predicted from readily available sensor data.
\end{abstract}

Index Terms: Predictive maintenance, Bleed of valve, Principle Component Analysis, Autoencoder, Aero derivative engines, Multi-Layer Perceptron.

\section{Introduction}

The demand of energy sources increases in the world every year. Even though, there is a strong shift toward energy generated by the renewable energy sources, the world still heavily relies on the oil and gas production. It should not be forgotten that the crude oil utilized not just for the creation of the oil by-products like diesel, kerosene but it is used for production of plastic, asphalts, food, furniture and many other things we use on daily bases.

Usually, middle range power generation units are being utilized on onshore and offshore oil and gas exploration and production facilities. Low operational and maintenance cost of such power generation solutions is becoming more and more important due to the fluctuating oil prices and strict rules for low carbon emissions.

Basically, the power generation package consists of the engine (internal or aero derivative), which either mechanically or aerodynamically (by a mean of exhaust flow) spins the turbine. The turbine outer shaft is either mechanically connected to the speed up / down gearbox for the optimum torque and speed or directly to the alternator for power generation. The power generation units would convert the chemical energy of burning fuel to the mechanical energy of the rotation and further to the electrical energy of the power generation.

The successful power generation package operation requires many auxiliary pieces of equipment like air filtration for combustion and cooling, oil for lubrication and cooling of the rotating parts, hydraulic for actuators movements etc. The operation of the engine employs a great number of instrumentation measurements devices, actuators and various programmable controllers.

The cost of operating power generation units is high due to continuous engine unitization, ageing and wear of different parts. Also, the loss of power generation on offshore platform or onshore terminal can potentially mean full loss of production and black out.

One of the approaches to reduce the operation and maintenance cost and increase package's availability and reliability would be to insure on utilization of the package in an efficient and safe way. Replacement of the wearing / ageing parts based on the operational data (predictive maintenance) rather than preventive (based on the service life) or run to the failure [1].

For this goal, one can utilize the RUM (Remote Unit Monitoring) facility with wear and failure prediction 
recognition logic to warn the maintenance team of the required maintenance. It should be also noted that some failures may exist on the engine for months before being identified and those failures can have further negative impact on overall engine performance. As one of the examples, the failure with engine BOV (Bleed of Valve) system, which leads to drop in engine efficiency and operation at higher exhaust temperature may be undetected for a quite long time and cause increased price of the engine planned overhaul. The difficulties to detect the bleed system failure due to complex relationship between various parameters, and this capability is out of the functional features for the package standard PLC (Programable Logic Controllers). At the same time, complex problems identification and failure prediction solutions are widely and successfully used in different industries such as aero [2-9], mining [10], and automotive [11].

After review of various publications, it was identified that the industrial power engines used in oil and gas industry are lagging with their technological advance in comparison to the mentioned above aero industry. To the limit of our knowledge, the utilization of predictive maintenance is not applied to industrial engines where the accepted approach is to consider BOV failures as part of life expectancy maintenance. This leads to suboptimal efficiency during operation and high maintenance costs.

Moreover, none of the bleed systems valves (starting or handlings) have feedback limit switches installed to confirm on the real valve movement either to open or close position, when commanded by PLC. The engines model RB211 currently in operation in Oil and Gas Terminal have a bad history, when the bleed valves MP6 (medium pressure stage 6) or HP6 (high pressure stage 6) failed to close at N_20 (specific engine calculated non dimensional speed used for BOV control) higher than 343 due to defective valve's internals or control air supply. The problem with open bleed valve cannot be detected by PLC as these involve many parameters and they are engine and ambient depending values. In other words, the attempts to put in place a PLC logic to detect the bleed system valve stuck in open position were made but this was proven unsuccessful.

The pneumatic valves do not contain the feedback limit switches due to the construction of the valves and its location on the engine (potential for dropping inside the engine and causing the major engine failure), hence these modifications physically could not be implemented.

The objective of this work is to develop an algorithm that can predict aero derivative engines bleed system failures using measurements obtained from existing sensors without the need to add any supplementary sensor.

In this paper we propose to use the readily available operational data from a Supervisory Control and Data Acquisition (SCADA) software. Available data is analysed, and a set of variables are selected as potentially relevant for fault diagnosis. Then, different machine learning algorithms are evaluated based on their ability to identify faults when trained on a limited amount of data. Feedforward Neural Networks, Linear Regression, and Random Forest algorithms are compared. Furthermore, two commonly used dimensionality reduction techniques, namely Principle Component Analysis (PCA) and Autoencoder (AE), are employed in order to improve the ability of the model to generalize.

One of the main challenges in using data analysis for fault identification is the limited amount of failure data. In fact, an engine usually operates under normal conditions and only occasionally fails. In this paper we propose to generate training data representing failure from a forced valve test. We show that a model trained on such "artificial" failure data is able to detect faults during operations.

The main contributions of this paper could be summarized as:

- A machine learning approach for BOV fault identification that can generalize from a limited amount of training data.

- $\quad$ A strategy to generate training data when failure data is not available.

The remainder of the paper is organized as follows: Section two summarizes the related literature on fault diagnosis in relevant areas. Section three provides an overview of the engine and the type of failure we are interested in. Section four describes the collected data and features. Section five presents the proposed approach and Section six the experimental results. Finally, we conclude in Section seven.

\section{Related Work}

The application of machine learning for failure identification and prediction is common in different areas. The most relevant area to our work is the airplanes industry where the engines used are similar to the ones we are considering in this paper.

A data-driven prognostic learning model was proposed for the aircraft bleed air valves failure identification in [2]. The study offered the data collection from the aircraft on board data recorder with further analysis and application of the complex statistics to gain the knowledge of the equipment reliability and necessary maintenances without really considering the engineering principles or physics. The used data-driven method was based on the two types of the data analysis, health monitoring and the past failure times. The health monitoring data represented the diagnostic fault messages collected form the 39 commercial aircraft control systems. The collected data was analysed to identify the times, when the bleed system had fault messages and replacements. Then, the data was tried on seven machine learning algorithms, LM (Linear regression), Bayes (Bayesian generalized linear model), KNN (k-nearest neighbour regression), 
NN (Neural Network) and few others. The study showed that the data driven prediction algorithm for the bleed system fault identification and parts replacement performs better than the traditional part service life expectancy maintenance approach.

The authors in [3] describe the application of Deep Learning techniques to the Aircraft faults detection, maintenance and repair. The paper describes the utilized deep learning architectures such as, Long Short-Term Memory (LSTM), Deep Belief Network (DBN), Deep Autoencoders (DAE) and Convolutional Neural Networks (CNN) and suggests the best application of each model. LSTM was able to identify the long-term data dependencies and it was used in the aero industry engines for the faults identification and estimation of the useful life for the various parts. DBN was suggested for utilization in the aero engines for faults diagnostics, classification and defects identification in the engine critical auxiliaries like a fuel system. DAE was used for aero planes structural integrity monitoring, and engine failure detections. Although the study is not directly related to bleed valves failure identification, it shows that different types of faults could be effectively detected using machine learning.

Another study, [4], reflects the successful machine learning model used for the aero engines bleed valves maintenance. The information from Singapore Airlines planes were collected from the QAR (Quick Access Recorder) as input data. As the QAR records many datasets, the collected data was processed to use the sets of data only in concerns for the engine bleed system monitoring, such as engine bleed system pressures and temperatures. Then, the airline maintenance records were compared against the data to identify if the bleed system was working correctly or with defects. Once identified, the data covering few months were classified as good or bad and applied into the machine learning classifier for the model training. The study used machine learning Gradient Boosting Machines (GBM) classifier. The model was trained and later validated on the data from the QAR. The model was able to identify the bleed valves failure with accuracy of $90 \%$. The $10 \%$ of missed accuracy was investigated and concluded that those failures did not have necessary measurement sensors inputs into the QAR for the model to identify these failures scenarios. As a result, the research study stated that the additional sensory must be used and configured in the QAR for the full coverage of the bleed valves failure identification by the used machine learning techniques.

The authors in [5] presented the use of derivation of the diagnostic observations and diagnostic model for the aero engine complex systems bleed off valves health monitoring. The study was focused on establishing the machine learning algorithm for early fault detection based on the recorded data form the built-in engine sensors. The method was developed and validated utilizing over 100000 of signals readings obtained from the real aircraft commercial flights. Naïve Bayes network and the decision trees were used as models. The testing with the aircraft real data reflected the model high accuracy over $99 \%$.

A supervised machine learning approach for fault identification on the engine bleed valve system was shown to be successful in [6]. In the study, the three different major failure modes were identified by a use of the computer model simulations. The failures were: valve chamber leak, piston excessive friction and valve return spring degradation. Support Vector Machine (SVM) and Classification Tree were used as models. Both machine learning algorithms were capable to identify the bleed valve problem but the SVM accuracy was slightly better.

The study described in [7] discusses the application of SVM to the problem of the aircraft's maintenance and faults prediction. In the work, the flight data from various aircrafts were collected together with the maintenance logs. The examined system was the jet engine bleed air system valves, and the recorded parameters were compressor delivery pressures, engine speeds, temperatures. The recorded data were pre-processed and compared to the planes maintenance logs to identify the condition for the data to formulate the output as healthy (the system performs as expected) or unhealthy (probability of the bleed system failure). The carried work concluded that the faults could be easily identified by the SVM classifier.

In [8] UKF (Unscented Kalmar Filter) were proposed for the engine bleed air system components and sensors failure detection. The described method consists of two UKFs, one for the bleed system parts like solenoids, tubes, pneumatic actuators and the second filter for the measurement sensors like pressure and temperature probes. The aero engine bleed air system control dynamics described and used by the nonlinear state space equations.

In [9] DAE was used on raw engine data from the various measurement sensors to develop a model to identify engine anomalies by analysing the reconstruction error.

To sum up the literature review, the remote data collection and analysis to predict and monitor the equipment condition has been successfully utilized in aero industry. The data driven online monitoring solutions are well applied to the aircrafts bleed valves system with various machine supervised or unsupervised learning algorithms but there is little available information or knowledge on the use of remote diagnostics with machine learning for the industrial engines. The aim of this paper is to propose a data-driven approach for bleed valve system failure prediction in industrial engines using readily available sensor data. 


\section{Aero-derivative Engines and Bleed Valve Systems}

In this section we describe the aero-derivative engines that are the object of this work.

\subsection{Aero-derivative engines}

The main power generation fleet is operated by BP (British Petroleum) and located on the onshore terminal at the Caspian seacoast. The total fleet comprises of six aero derivative engines.

The engine comprises of the intake plenum, compressor, combustion chamber, turbine and exhaust section, as shown in Fig1.

The inlet plenum directs the air flow into the compressor, where the air being compressed prior entering the combustion chamber. The fuel gas being added through the injectors and mixes with the air form the compressor. Then, the mixer being ignited and directed to the turbine to strikethrough blades, what in turn spins the compressor (mechanically coupled on the same shaft). The exhaust flow would flow through separate turbine (not mechanically coupled), which rotates the AC generator for power generation.

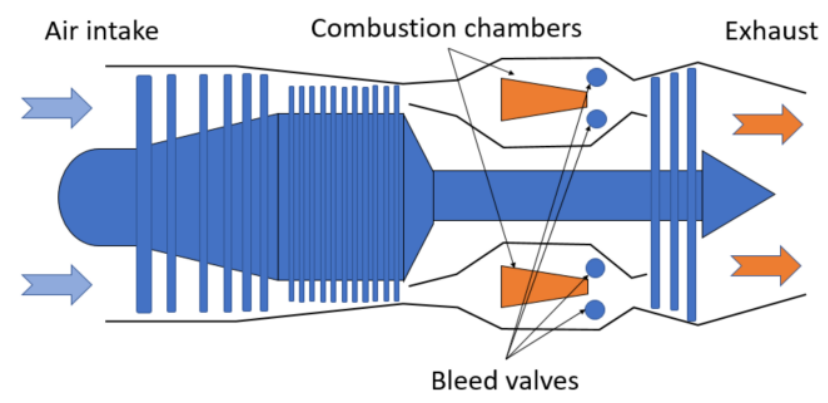

Fig.1. Aero-derivate engine and the position of bleed valve systems.

There are many auxiliary systems, which support the power generator operation and they were already described in the above sections. Also, as was already stated the availability and reliability (A\&R) of the generators declines as the equipment gets older and we are looking to reduce the cost of maintenance together with increasing the generators A\&R.

For the solution, we are proposing to transfer the data from the main power generator units to the remote diagnostic facility. The special models (such as Feedforward Neural Network) would analyse the data and generate the reports to advise on the predictive maintenance. The reports would be used for the site operational team.

\subsection{Engine bleed valves system and its control logic}

The engine bleed system's basic function is to prevent gas generator compressor surge and stall by venting an excess air using BOV during starting and stopping to improve the operational efficiency at low load conditions.

There are a few bleed valves with descriptions as below:

- 2 off MP6 handling bleed valves spilling MP stage 6 air from intermediate pressure compressor and scheduled to open and close by the ECS (Engine Control System) in accordance with a corrected M.P. compressor speed. The engine PLC would command the muscle airline control solenoid to open or close, hence introducing or shutting off the air line to the pneumatic valve to make it open or closed.

- 1 off HP6 handling bleed valve spilling air scheduled to open and close by the Engine Control System in accordance with a corrected M.P. compressor speed, the same logic as described for MP6 control. The ECS commands to close valves at a non-dimensional MP speed (N_20) of $343+/-1$ rising and open them at 334 $+/-1$ falling.

- $\quad 3$ off starting bleed valves spills air during the starting cycle only and controlled by the engine internal air.

The basic operation of the control logic can be summarized as follows:

- All BOV stay open if the engine operates at a speed lower than a threshold (usually this is the condition during engine start up or shut down)

- All BOV stay closed while engine operates at the rated power (the engine operates at a speed higher than a threshold).

As was described above, the three starting BOV are controlled by the engine internal air pressure (pneumatic control only). So, as the engine speed increases, the compressor pressure overcomes the mechanical settings of the starting BOV spring force and causes them to close. The other set of valves MP6 and HP6 handling valves are being 
commanded to close / open depending on the engine non-dimensional speed, where the PLC opens or closes the control air supply solenoid, which in term causes the MP6 and HP6 to operate. The engine air and the internal spring force keep the valves open and the valves close when the solenoid shuts off the control air supply, so the internal engine air overcomes the valve spring force.

None of the bleed system valves (starting or handlings) have feedback limit switches installed to confirm on the real valve movement either to open or close position, when commanded by PLC. The engines have a bad history, when the bleed valve MP6 or HP6 failed to close at N_20 higher 343 due to failure of the valve's internals or defective control air supply caused by stack solenoid. The problem with open bleed valve cannot be detected by PLC as these involve many parameters and they are engine and ambient depending values. In other words, the attempts to put in place a PLC logic to detect the bleed system valve stuck open position were made but this was proven unsuccessful.

The pneumatic valves do not contain the feedback limit switches due to the construction of the valves and its location on the engine (potential for dropping inside the engine and causing the major engine failure), hence these modifications physically could not be implemented.

\section{Data Collection}

The data was collected from running engines between 1st of January till 3rd of April 2020. During the period four real failure events occurred.

Moreover, to simulate failure conditions, a stroke test was carried out and nineteen failure events were generated, and the corresponding measurements were taken. The tests were performed on five units. The testing consisted of forcing open the air control solenoid for the duration of ten seconds in five cycles. The solenoid forcing would cause engine medium and high pressures compressors BOV to open, like the BOV system failure.

It is important to note that it is easier to record normal operational data compared to failure data. In total 10162 data points were collected. Each data point corresponds to a vector of measurement at a certain time step. The distribution of the collected data is shown in Table 1 below.

Table 1. The distribution of the collected data.

\begin{tabular}{cccc}
\hline & Real & Stroke & Total \\
\hline Normal (output $=1)$ & 4803 & 4034 & 8837 \\
Failure (output $=0)$ & 1285 & 40 & 1325 \\
Failure events & 4 & 19 & 23 \\
\hline
\end{tabular}

\subsection{Feature selection}

Based on operational experience and a preliminary analysis of a BOV failure event that occurred in November 2019 a list of parameters that could be measured was defined. These parameters constitute the features describing a data point or a sample. The parameters are summarized in Table 2 below.

Table 2. Selected measurement parameters.

\begin{tabular}{|c|c|c|c|}
\hline Tag number & Description & Unit & Update rate \\
\hline EGH_455 & Engine exhaust profile temperature (17c Thermocouples) & $\operatorname{Deg} C$ & Every 3 seconds \\
\hline P_20 & Medium ratio compressors delivery pressures & Barg & Every 3 seconds \\
\hline P_40 & High ratio compressors delivery pressures & Barg & Every 3 seconds \\
\hline$N 20$ & Medium compressor speed & $R P M$ & Every 3 seconds \\
\hline N40 & High compressor speed & $R P M$ & Every 3 seconds \\
\hline$E G T \_P 455$ & Exhaust pressure & Barg & Every 3 seconds \\
\hline$T 1$ & Ambient temp & Deg $C$ & Every 3 seconds \\
\hline$F G R$ & Fuel gas regulated press. & Barg & Every 3 seconds \\
\hline$F G M$ & Fuel gas manifold press. & Barg & Every 3 seconds \\
\hline$M W$ & Engine output power & Mega Watts & Every 3 seconds \\
\hline$T 20$ & Engine medium press. Compressor air flow temp & $\operatorname{DegC}$ & Every 3 seconds \\
\hline$T 40$ & Engine High press. Compressor air flow temp & $\operatorname{DegC}$ & Every 3 seconds \\
\hline
\end{tabular}

\subsection{Feature selection justification}

A feature that is suitable for BOV failure prediction should be readily available, i.e. sensor data already managed by the SCADA system, and should be discriminant, e.g. correlates with failure events. In this section we provide a justification for the selection of the proposed features in Table 3.

\section{EGH_455}

The data of interest for our work would be EGT (exhaust gas temperature). If the trends show the EGT for the 
engine increasing and other parameters such as P20 and P40 compressor pressures are decreasing, or a rapid deceleration of the output power, then gas generator turbine bleed system deterioration can be suspected. An increase of $10-12^{\circ} \mathrm{C}$ from the normal running condition would indicate that the engine requires maintenance. It can be indicating either bleed valve stuck in partially or fully open position, when commanded to be closed.

\section{P20 and P40.}

Effectively, opening of BOV and uncontrolled bleed of the air would lead to the rapid decrease of the pressure at the sensors measuring points. This happens as the commonly delivered air flow from engine compressors would have an alternative route to flow.

\section{N20 and N4O}

The engine speeds would initially decrease because of the loss in the engine mass air flow delivered to the combustion chamber for conversion from chemical energy of burning into mechanical energy of rotating. The speed would recover to some degree as the engine PLC would command electrical metering valves to put more fuel and IGV to let higher air flow.

\section{EGT_P455}

Less energy and air flow to combustion chamber would result in loss of exhaust pressure sensed at rear side of the engine.

\section{$T 1$}

This parameter can be classified as independent from the engine anti-surge system and more relative to the ambient conditions (ambient temperature and pressure). By inputting these data, we hope the model would auto compensate for the changes in ambient values.

\section{FGR, FGM}

From the captured data reflecting the real case with BOV failure, the event caused changes in the fuel gas pressures. The explanation would come from the fact, as air delivered to combustion reduces and engine overall power decreases, the engine PLC commands fuel motorized valve to open more to compensate for this condition. As the motorize valve opens, the FGR regulated pressures decreases and FGM would increase.

\section{T20 and $T 40$}

Engine air temperatures delivered by the medium and high pressures compressors can give a good correlation with engine power. If the temperatures decrease for the given power with the same ambient conditions, then this gives a good guide of the engine bleed system deterioration.

\section{Engine output power $(M W)$}

It is obvious, with decrease in engines speeds, less air into combustion chamber would lead to the power loss. As the demand for the power level dictated by the common grid, and if the engines operate in power sharing mode, then the other engine running in parallel would compensate for the loss. The power output on the engine with defective BOV would recover to some degree but not fully.

\section{Proposed Approach}

The features described in the previous section could be used to constitute the input feature vector for a machine learning model. The machine learning model's output would be a real number between zero and one, where zero corresponds to failure condition and one to normal condition. Consequently, the machine learning model would output a probability of normal condition. It is preferable to use a regression model that produces probability of normal condition rather than a classification model that provides a class (failure, normal). In fact, as shown in the literature review section, it is common for a classification model to produce results that are close to $100 \%$ accuracy. This would make it difficult to compare different alternatives. However, in a regression problem the numerical nature of the output will allow us to compare the alternatives. Consequently, we propose to develop a regression model that produces as an output a value between zero and one corresponding to failure and normal conditions respectively.

Different machine learning models could be used. In this paper we evaluate three machine learning models, namely Artificial Neural Networks, Linear Regression, and Random Forest. We propose also to evaluate the effect of dimensionality reduction on the input feature vectors on the performance.

\subsection{Artificial Neural Networks}

Artificial Neural Networks (ANN) are a family of machine learning algorithms that inspired by biological systems. They are used for classification and regression [12]. An ANN is composed of a set of basic elements, the neurons, that 
are connected via links called synapses. The connection between neurons has weight called synaptic weight. Within each neuron, a relatively simple operation is carried out. It is typically a function applied on the weighted sum of all its inputs. The neuron then sends the output to other neurons it is connected to. Neurons are organized in layers. The input layer is the one that receives the feature vector, the output layer is the layer that produces the output, and any layer in between is called a hidden layer [13]. In our case the output layer contains one neuron corresponding to the probability of normal condition as shown in Fig.2. below.

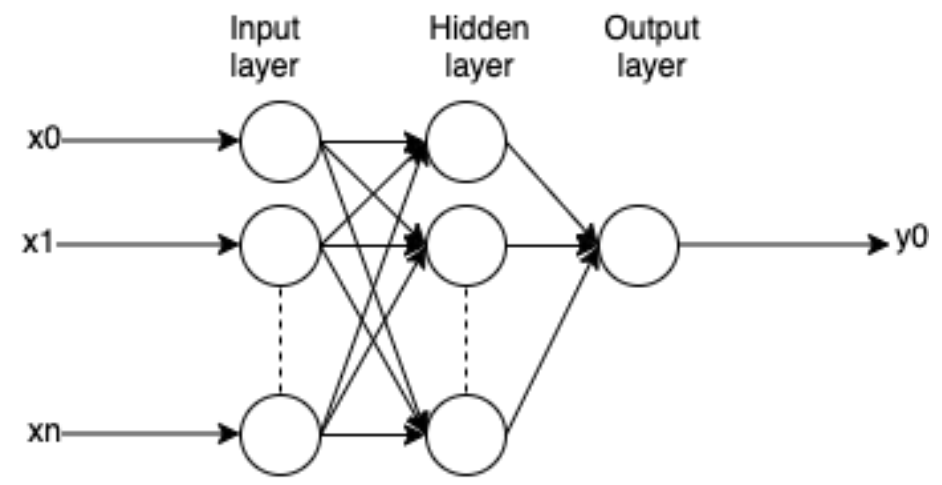

Fig.2. Artificial Neural Network architecture

\subsection{Linear Regression}

Linear Regression is a machine learning technique where a dependent variable, the output, is calculated as a weighted sum of independent variables, the features [14]. Formally, a Linear Regression model represents the output $y$ as a function of independent variables $x_{i}$ as follows:

$$
y=\beta_{0}+\sum_{i=1}^{n} \beta_{i} x_{i}
$$

Where $\beta_{i}$ are suitable weights.

\subsection{Random Forest}

Random Forests are models used for both classification and regression problems. They combine multiple Decision Trees that apply a set of if-then rules on input data, the features, in order to provide a class or a value for the output [15].

\subsection{Feature correlation analysis}

It is reasonable to assume that the features described in the previous section are correlated. To verify this assumption, we did a correlation analysis on all the of the collected data described in the data collection section. As shown in the Table 3 below, a high correlation between the features exists.

Table 3. Feature correlation.

$\begin{array}{ccccccccccc} & \text { N20 } & \text { N40 } & \text { P_20 } & \text { P_40 } & \text { EGT_P455 } & \text { FGM } & \text { FGR } & \text { T40 } & \text { MW } & \text { T20 } \\ \text { N20 } & 1.00 & 0.98 & 0.94 & 0.94 & -0.17 & -0.35 & 0.86 & 0.93 & 0.96 & 0.17 \\ \text { N40 } & 0.98 & 1.00 & 0.92 & 0.92 & -0.15 & -0.38 & 0.83 & 0.93 & 0.93 & 0.27 \\ \text { P_20 } & 0.94 & 0.92 & 1.00 & 0.99 & 0.01 & -0.30 & 0.78 & 0.83 & 0.99 & -0.04 \\ \text { P_40 } & 0.94 & 0.92 & 0.99 & 1.00 & 0.04 & -0.25 & 0.77 & 0.81 & 0.98 & -0.04 \\ \text { EGT_P455 } & -0.17 & -0.15 & 0.01 & 0.04 & 1.00 & 0.64 & -0.60 & -0.47 & -0.05 & 0.03 \\ \text { FGM } & -0.35 & -0.38 & -0.30 & -0.25 & 0.64 & 1.00 & -0.59 & -0.58 & -0.36 & 0.02 \\ \text { FGR } & 0.86 & 0.83 & 0.78 & 0.77 & -0.60 & -0.59 & 1.00 & 0.95 & 0.81 & -0.03 \\ \text { T40 } & 0.93 & 0.93 & 0.83 & 0.81 & -0.47 & -0.58 & 0.95 & 1.00 & 0.86 & 0.19 \\ \text { MW } & 0.96 & 0.93 & 0.99 & 0.98 & -0.05 & -0.36 & 0.81 & 0.86 & 1.00 & 0.00 \\ \text { T2 } & 0.17 & 0.27 & -0.04 & -0.04 & 0.03 & 0.02 & -0.03 & 0.19 & 0.00 & 1.00\end{array}$

The high correlation between features indicates that an eventual dimensionality reduction could be applied. In this paper we evaluate two reduction methods, Principal Component Analysis (PCA) and Multi-Layer Perceptron (MLP) Autoencoder (AE). 


\subsection{Principle Component Analysis}

Principle Component analysis (PCA) is a technique used to reduce the dimensionality of feature vectors in machine learning [16-18].

PCA maps a feature vector of dimensionality $d$ into a feature vector of dimensionality $n$ where $n<d$. Each dimension in the PCA-transformed vector is a linear combination of the original dimensions and is called a principle component. The operation can be represented as mapping from an original vector $\mathrm{x}$ to a transformed one PC as follows:

$$
\begin{gathered}
x=\left[x_{1}, x_{2}, \ldots, x_{d}\right] \rightarrow P C=\left[P C_{1}, P C_{2}, \ldots, P C_{n}\right] \\
P C_{i}=\sum_{j=1}^{d} a_{j i} x_{j}
\end{gathered}
$$

Where $a_{j i}$ are suitable weights.

Principle components are ordered by their description of the variance of the data in a dataset. For example, the first principle component $\mathrm{PC}_{1}$ explains more variance in the data than the second principle component $\mathrm{PC}_{2} \cdot \mathrm{PC}_{2}$ explains more variance than $\mathrm{PC}_{3}$, etc. Therefore, the first few principle components can describe the grand majority of variance in a dataset. Consequently, when applying PCA on a dataset, one can select a limited number of principle components in the transformed feature vector and prune the rest.

\subsection{Autoencoder}

An Autoencoder (AE) is a method used for dimensionality reduction [19]. It employs an Artificial Neural Network (ANN) that is trained to produce an output equals to the input. The ANN contains a hidden layer with fewer number of neurons than the input neurons. Once the ANN is trained on a dataset, it is used to map an original feature vector into a transformed one by taking the output of the hidden layer when the original feature vector is introduced as the input to the neural network as shown in Fig. 3.

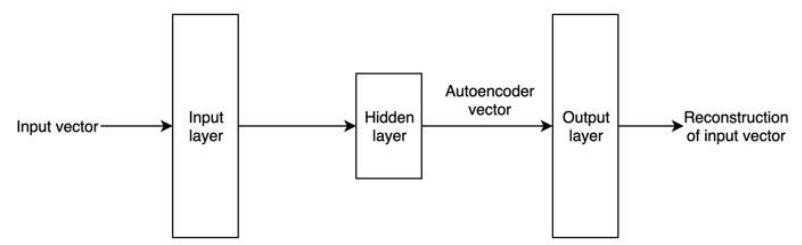

Fig.3. Autoencoder for dimensionality reduction.

\section{Experimental Results}

The collected data was split into a "Real 1", "Real 2", and "Stroke" datasets. The Table 4 below shows the distribution of data.

Table 4. Experimental data distribution.

\begin{tabular}{cccc}
\hline & Real 1 & Real 2 & Stroke \\
\hline Normal (output $=1)$ & 3615 & 1188 & 4034 \\
Failure (output $=0)$ & 19 & 1266 & 40 \\
Failure events & 1 & 3 & 19 \\
\hline
\end{tabular}

Three experiments were carried out to evaluate the model:

- Experiment 1 (Exp. 1): the model was trained on "Real 1" dataset and tested on "Real 2" dataset.

- Experiment 2 (Exp. 2): The model was trained on "Real 1" dataset and tested on "Stroke" dataset.

- Experiment 3 (Exp. 3): The model was trained on "Stroke" dataset and tested on "Real 1" and "Real 2" datasets.

Exp. 1 and Exp.2 evaluate the capability of the model to generalize from a small training dataset. Exp. 3 simulates the situation where one explicitly generates data for training if no previous failure events have occurred. In fact, in real world applications, failures are much less common than normal operation. Hence, gathering training data that covers failure events is difficult in practice. Hence, one needs to be able to train a model on a limited amount of data. Moreover, one must be able to generate suitable training data if no or insufficient failure events have occurred. Exp. 1 \& Exp. 2 are 
relevant to the former situation and Exp. 2 \& Exp. 3 to the latter.

The experiments were carried out for the full feature vector and after the application of the PCA and Autoencoder (AE) dimensionality reduction techniques.

To evaluate the models, we used the correlation coefficient $\mathrm{R}$, and the relative absolute error RAE.

$$
\begin{gathered}
R=\frac{\sum_{i=1}^{n}\left(T_{i}-\bar{T}\right)\left(P_{i}-\bar{P}\right)}{\sqrt{\sum_{i=1}^{n}\left(T_{i}-\bar{T}\right)^{2}} \sqrt{\sum_{i=1}^{n}\left(P_{i}-\bar{P}\right)^{2}}} \\
R A E=\frac{\sum_{i=1}^{n}\left|P_{i}-T_{i}\right|}{\sum_{i=1}^{n}\left|T_{i}-\bar{T}\right|}
\end{gathered}
$$

Where $P_{i}$ is the model's output, $\bar{P}$ is the mean of the model's outputs, $T_{i}$ is the target output, $\bar{T}$ is the mean of the target outputs.

\subsection{Full feature vector}

In this experiment we used the full feature vector without any dimensional reduction applied. Three different classifiers were evaluated: Feedforward Neural Network, linear regression, and random forest. The Feedforward Neural Network's architecture is a Multi-Layer Perceptron (MLP). We evaluated two versions, one MLP with one hidden layer and one MLP with two hidden layers.

Table 5 shows the obtained results when using the full feature vector. In this table, MLP 10H is the MLP with one hidden layer and MLP10H10H is the MLP with two hidden layers. Each hidden layer contains ten hidden neurons. The number of hidden neurons was selected after a development phase that showed ten hidden neurons provide the best performance.

The results show the correlation coefficient and the relative absolute error.

\begin{tabular}{|c|c|c|c|c|}
\hline & MLP10H & MLP10H10H & Linear Regression & Random Forest \\
\hline Exp. 1 & $0.99(10 \%)$ & $0.99(4 \%)$ & $0.99(13 \%)$ & $0.97(46 \%)$ \\
\hline Exp. 2 & $0.99(24 \%)$ & $0.99(25 \%)$ & $0.99(61 \%)$ & $0.99(35 \%)$ \\
\hline Exp. 3 & $0.99(2 \%)$ & $0.99(3 \%)$ & $0.99(19 \%)$ & $0.99(17 \%)$ \\
\hline Average & $0.99(12 \%)$ & $0.99(11 \%)$ & $0.99(31 \%)$ & $0.98(33 \%)$ \\
\hline
\end{tabular}

Table 5. Experimental results showing the correlation coefficient and the relative absolute error in percentage.

As seen in the table above, MLP provides better results than Linear Regression and Random Forest for both criteria, correlation coefficient and mean absolute error. On average, MLP 10H gives a mean absolute error of $12 \%$ and a correlation coefficient 0.99 compared to $11 \%$ and 0.99 for MLP $10 \mathrm{H} 10 \mathrm{H}$. The two hidden layer MLP is therefore providing the best results.

\subsection{Dimensionality reduction}

As discussed earlier, two dimensionality reduction techniques are proposed: PCA and Autoencoder. Using both reduction methods the feature vector's dimensionality was reduced to four. In the case of PCA this covers $98 \%$ of variance. For comparable results we selected the same dimensionality when using Autoencoder. Interestingly, the performance of Linear Regression and Random Forest considerably degraded. Consequently, only MLP results are shown below in Table 6.

Table 6. Dimensionality reduction experimental results showing the correlation coefficient and the relative absolute error.

\begin{tabular}{ccccc}
\hline & PCA & PCA MLP10H10H & Autoencoder MLP10H & Autoencoder MPL10H10H \\
\hline MLP10H & $0.97(15 \%)$ & $\mathbf{0 . 9 9 ( 3 \% )}$ & $0.99(4 \%)$ & $0.99(2 \%)$ \\
Exp. 2 & $0.97(37 \%)$ & $\mathbf{0 . 9 9}(\mathbf{7 \%})$ & $0.93(146 \%)$ & $0.94(144 \%)$ \\
Exp. 3 & $0.99(1 \%)$ & $\mathbf{0 . 9 9 ( 1 \% )}$ & $0.98(5 \%)$ & $0.98(4 \%)$ \\
Average & $0.98(18 \%)$ & $\mathbf{0 . 9 9}(\mathbf{4 \%})$ & $0.97(52 \%)$ & $0.98(50 \%)$ \\
\hline
\end{tabular}


As show in Table 6, PCA performed better than the Autoencoder method with respect to the correlation coefficients and relative absolute errors. Moreover, MLP10H10H provides better results than MLP10H. Compared to the full feature vector, PCA with MLP $10 \mathrm{H} 10 \mathrm{H}$ improves the results. The average relative absolute error drops from $11 \%$ to $4 \%$.

It is worth noting that if the problem is converted into a classification problem where the classes are "failure" and "normal" by applying a threshold of 0.5 to the model's output, the results are $100 \%$ precision and $100 \%$ recall.

Moreover, the experimental results have demonstrated that there is no need to convert inputs into ambient corrected data. The inputs used in the experiments were not corrected to an ISO standard day i.e. $15^{\circ} \mathrm{C}$ ambient temperature and 14.696 psia (1.01325 bar) atmospheric pressure [20].

\subsection{Failure identification}

The experimental results suggest that an MLP with two hidden layers and dimensionality reduction using PCA is the most suitable approach. Hence, we propose the use of MLP10H10H after applying PCA transformation on the feature vectors.

Fig.4. and Fig.5. show the comparison between the model's output and the expected output for the stroke test data and Real 2 test data. As can be seen in the figures, the model correctly identifies all failure events with a comfortable margin even after being trained on one failure event only.

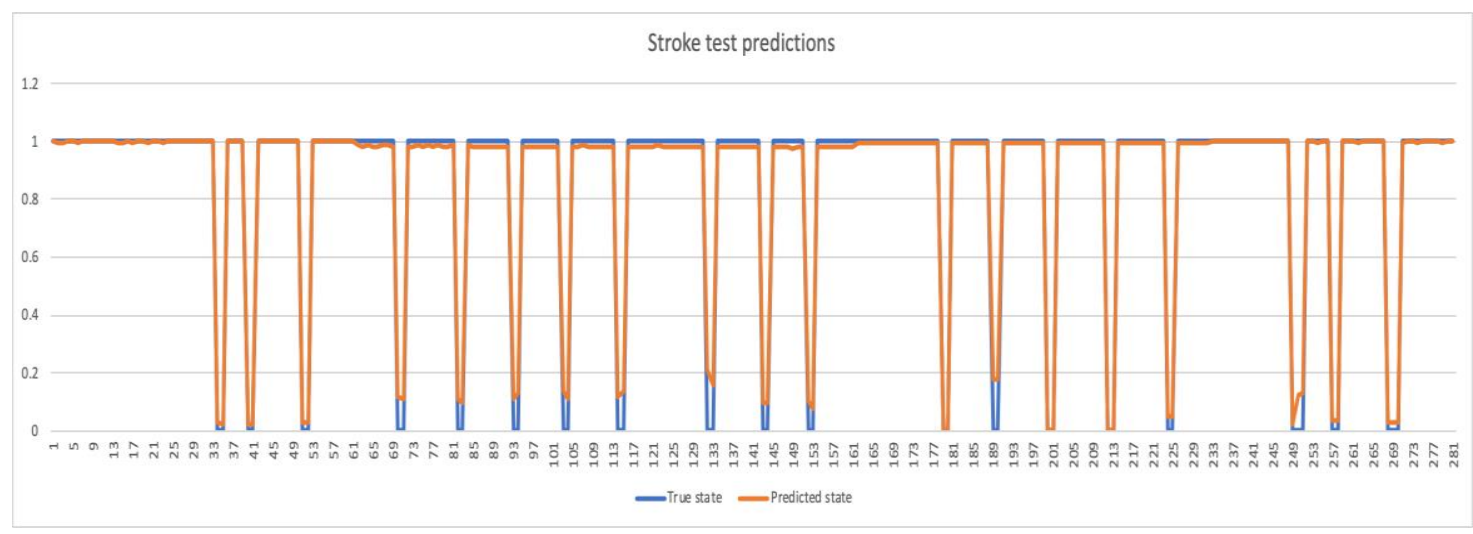

Fig.4. Model output for stroked data

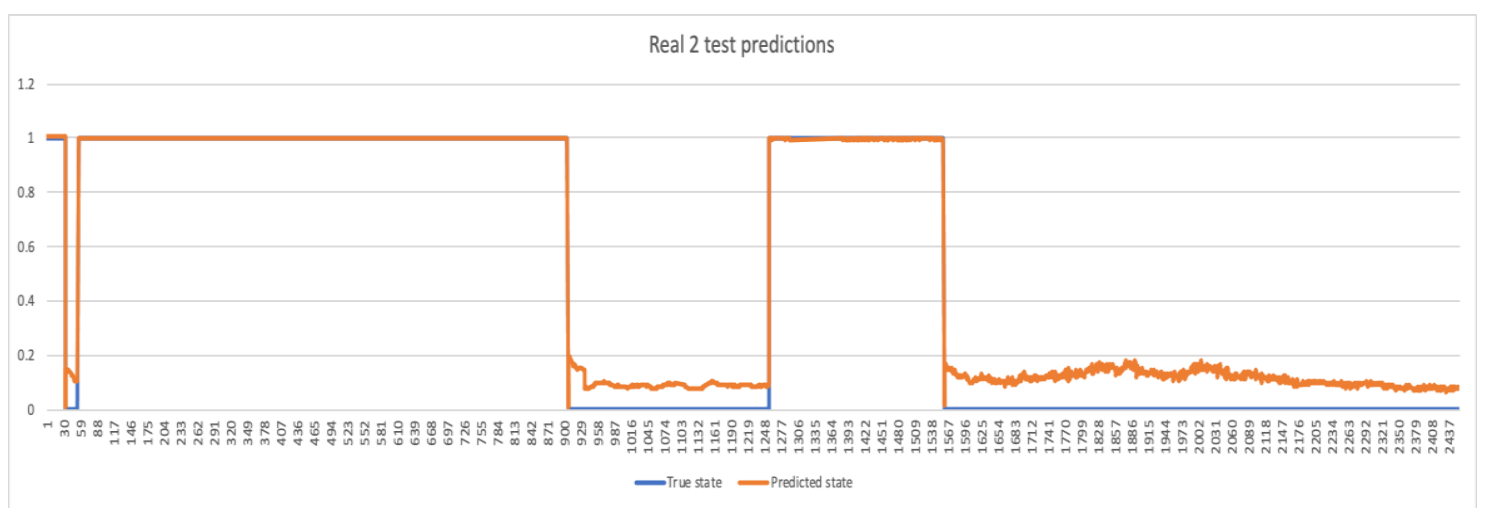

Fig.5. Model output for real data

\subsection{Discussion}

From the experimental results presented above showed that BOV failures could be effectively identified using readily available sensor data. A Neural Network with two hidden layers performed better than a Neural Network with one hidden layer. It also performed better than a Linear Regression and a Random Forest model. This suggests nonlinearities on the relationship between the variables used as features, the sensor data, and the probability of normal condition. Moreover, dimensionality reduction improved the results. This confirms that correlation exists between the initial features that were used. The results showed also that a model trained on stroke data, that simulate a failure, are effective in identifying real failures. This is particularly important for practical applications because failure data are not always available to train a model. One can hence develop a model and train it on stroke data instead. 


\section{Conclusion}

Aero derivative engines' anti surge system is classified as critical. Its correct functioning is required for the efficient and reliable operation. The failure of bleed of valve system leads to increasing operational cost, and negative environmental impacts as a result of increasing engine emissions. Early identification of bleed valve system failures is hence essential.

In this paper a data-driven bleed valve system failure identification approach is proposed. The developed model uses machine learning and readily available sensor data. Different machine learning algorithms and dimensionality reduction techniques are evaluated. The experimental results show that a Multi-Layer Perceptron with two hidden layers combined with Principle Component Analysis is able to identify bleed valve system failures in aero derivative engines. The model was evaluated on operational data from five units for over 60 days. The model proved to be able to generalize from a small amount of training data corresponding to one failure event. Moreover, we proposed a method to generate training data using stroke tests in case an engine has no previous failure data. When trained on the stroke test data, the model correctly identified real failure conditions. This paper showed that a machine learning model could be effectively applied to detect bleed valve system failures using readily available sensor data. The model is effective even when little or no failure data exists for training. Such model could be easily integrated into an existing infrastructure as it uses sensor data that are available in a SCADA system. Such a model could be employed in the context of a predictive maintenance approach. Moreover, the model could be re-trained regularly to include newly available data containing both normal operation and failure events.

The model's capability must be tested further however with various ambient and engine power output values. In particular, it is important to test the model on the long term, i.e. a model trained on past data and tested after one, two, three, etc. years on the same engine.

\section{References}

[1] Moubray, J. Reliability Centred Maintenance, 2nd ed., Elsevier, 1997.

[2] Marcia B., Ivo P. de Medeiros, Joao P., Malere, Helmut P., Cairo L., Nascimento Jr., and Elsa Henriques., 'A Comparison of Data-driven Techniques for Engine Bleed Valve Prognostics using Aircraft-derived Fault Messages'. in Proceedings of the 3rd European Conference on Prognostics and Health Monitoring (PHME), Bilbao, Spain, pp. 1-13, 2016.

[3] Divish, R, Herve P. M, Grazziela P. F. 'Deep Learning Approaches to Aircraft Maintenance, Repair and Overhaul'. In Proceedings of IEEE International Conference on Intelligent Transportation Systems, ITSC, Maui, USA, pp. 1-7, 2018.

[4] Ramasamy, S., Xue, Y., Phoon, R., Han, R., Low, N., Lim, C. S. 'Predictive Maintenance of the Aircraft Engine Bleed Air System Component' in Proceedings of the annual Conference of the Prognostics and Health Management (PHM), Philadelphia, USA, pp. 1-7, 2018.

[5] Wojtek P.K., David A., John V., Gary M., 'Health Monitoring for Commercial Aircraft Systems', in Proceedings of the 26th International Congress of The Aeronautical Sciences, pp. 1-8, 2008.

[6] Castilho, H.M., Nascimento, C.L., \& Vianna, W.O. 'Aircraft bleed valve fault classification using support vector machines and classification trees' in Proceedings of the annual IEEE International Systems Conference (SysCon), pp. 1-7, 2018.

[7] Moreira, R., Nascimento, J.C. 'Prognostics of aircraft bleed valves using a SVM classification algorithm' in Proceedings of IEEE Aerospace Conference Proceedings, pp. 1-8., 2012.

[8] Shang, L., Liu G. 'Sensor and actuator fault detection and isolation for a high performance aircraft engine bleed air temperature control system', in Proceedings of the 48h IEEE Conference on Decision and Control (CDC), Shanghai, China, pp. 4888-4893, 2009.

[9] Kishore K. R., Soumalya S., Vivek V., Michael Gi. 'Anomaly Detection and Fault Disambiguation in Large Flight Data: A Multi-modal Deep Auto-encoder Approach', in Proceedings of the Annual Conference of The Prognostics and Health Management Society, Vol. 2016.

[10] Juanli Li, Yang Liu, Jiacheng Xie , Menghui Li, Mengzhen Sun, Zhaoyang L. 'A Remote Monitoring and Diagnosis Method Based on Four-Layer IoT Frame Perception', IEEE Access, vol. 7, pp. 144324-144338, 2019.

[11] Mostafa T., Eman M., Mohammed D., and Mohamed E.. 'Remote Diagnosis, Maintenance and Prognosis for Advanced Driver Assistance Systems Using Machine Learning Algorithms', SAE International Journal of Passenger Cars - Electronic and Electrical Systems, pp. 115-122, 2016.

[12] Douglas T. Mugweni, Hadi Harb, " Neural Networks-based Process Model and its Integration with Conventional Drum Level PID Control in a Steam Boiler Plant ", International Journal of Engineering and Manufacturing (IJEM), Vol.11, No.5, pp. 1-13, 2021. DOI: $10.5815 / \mathrm{ijem} .2021 .05 .01$

[13] Haykin, S. Neural Networks, A Comprehensive Foundation, Prentice Hall, New Jersey. 1994

[14] T. Hastie, R. Tibshirani, M. Wainwright, Statistical Learning with Sparsity The Lasso and Generalizations, Taylor \& Francis Group, 2015.

[15] Breiman, L. Random Forests. Machine Learning (45), 5-32, 2001

[16] Tharwat, A.. 'Principal component analysis-a tutorial'. International Journal of Applied Pattern Recognition, vol. 3, pp. 197$240,2016$.

[17] Howley, T., Madden, M. G., O'Connell, M. L., \& Ryder, A. G. 'The effect of principal component analysis on machine learning accuracy with high dimensional spectral data' in Proceedings of the International Conference on Innovative Techniques and Applications of Artificial Intelligence, London, UK, pp. 209-222, 2005. 
[18] Deegalla, S., \& Bostrom, H. 'Reducing high-dimensional data by principal component analysis vs. random projection for nearest neighbor classification' in Proceedings of the 5th International Conference on Machine Learning and Applications (ICMLA'06), Orlando, USA, pp. 245-250, 2006.

[19] Wang, Y., Yao, H., \& Zhao, S. 'Auto-encoder based dimensionality reduction', Neurocomputing, vol. 184, pp. $232-242$.

[20] Volponi, A. J. (1999), 'Gas Turbine Parameter Corrections' ASME Journal of Engineering for Gas Turbines and Power, vol. 121, pp. 613-621, 2016.

\section{Authors' Profiles}

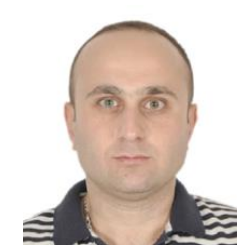

Khalid Salmanov Khalid Salmanov. Khalid Salmanov was born in Baku, Azerbaijan on $14^{\text {th }}$ of February 1982. He obtained his bachelor's degree in Oil and Gas Wells Driving in 2002 from the Azerbaijan State Oil Academy in Azerbaijan. Then, he decided to deepen his knowledge in the oil industry hence he enrolled and graduated his master's degree in Environmental Protection Measures Applied in the Oil Industry in 2004 in a cooperation with Azerbaijan State Oil Academy (Azerbaijan), University of Nizza (France), and University of Geneva (Italy). In 2004 he was employed by Rolls-Royce Energy Azerbaijan and worked as Control Engineer. During his employment with Rolls-Royce, Khalid gained a technical diploma from the Cleveland Institute of Electronics INC, Cleveland, Ohio USA in the faculty of Industrial Electronics with PLC Technology. He worked for Rolls-Royce over 8 years and his last position when he left the company was Automation Manager for Central Asia Region. Later in his career he joints British Petroleum (BP) as Senior Instrumentation and Control Engineer based in Azerbaijan. In 2020 he obtained Master Engineer degree in Industrial Automation with Institute of Engineering and technology, Perth, Australia. Currently, Khalid is working for Croda Europe Limited as Instrumentation and Control Engineer at the chemical production plant based in the United Kingdom. Khalid also is a Chartered Engineer with Institute of Engineering and Technology, UK.

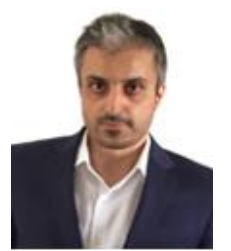

problems.

Hadi Harb Hadi Harb holds a MEng (2000) in electrical-electronic engineering from the Lebanese University. He earned his MSc in 2001 and PhD in 2004 both in computer science from the Institut National des Sciences Appliquées INSA Lyon France, and the Ecole Centrale de Lyon France, respectively. In 2004 he joined Centrale Lyon Innovation SA as a research engineer. In 2006 he founded and managed Ghanni, a company specialised in multimedia content recommendation and identification. Several European radio stations and websites licensed Ghanni's music recommendation technology. In 2015 he restructured Ghanni to transform it into a consultancy company in the domain of Artificial Intelligence and joined the Engineering Institute of Technology, Australia, as a lecturer. His current research interests are in the use of Artificial Intelligence techniques to solve industrial

How to cite this paper: Khalid Salmanov, Hadi Harb, "Data Analysis for the Aero Derivative Engines Bleed System Failure Identification and Prediction", International Journal of Intelligent Systems and Applications(IJISA), Vol.13, No.6, pp.13-24, 2021. DOI: $10.5815 /$ ijisa.2021.06.02 